\title{
HEPATOTOXICIDADE DO PARACETAMOL E FATORES PREDISPONENTES
}

\author{
Luciana Vilar Torres ${ }^{\text {* }}$ \\ Patrícia da Silva Oliveira" \\ Cibério Landim Macêdo III \\ Thaísa Leite Rolim Wanderley ${ }^{\text {IV }}$
}

\begin{abstract}
RESUMO
Os analgésicos não opioides constituem uma classe de medicamentos muito presentes em casos de intoxicação, sendo o paracetamol relevante devido suas características hepatotóxicas. Por ser um medicamento de venda livre, sua fácil aquisição e o pouco conhecimento sobre os efeitos prejudiciais ao organismo contribuem para o aumento de intoxicações, principalmente em crianças. O paracetamol é considerado hepatotóxico dependente de dose podendo gerar lesão de hepatócitos, através de mecanismos independentes, ou associados entre si. O objetivo desse estudo consiste em investigar os principais fatores que predispõem a hepatotoxicidade do paracetamol e seu mecanismo de hepatotoxicidade, tendo em vista o seu perfil de utilização, por meio de revisão bibliográfica de caráter exploratório e descritivo, através de pesquisa em livros e artigos científicos, realizada durante o período de julho e dezembro de 2017. A busca de artigos foi efetuada nas bases eletrônicas de dados Scielo, Science Direct, PubMed, Lilacs e Google Acadêmico. Na estratégia de busca, foram inicialmente avaliados os artigos por meio da análise dos títulos e resumos e, no total, a análise foi composta por 19 artigos. Alguns fatores podem predispor uma pessoa à hepatotoxicidade por paracetamol como medicamentos que inibem enzimas, jejum prolongado, etilismo, polimorfismo de genes, idade, entre outros. É importante que os profissionais envolvidos com medicamentos conheçam as características que o paracetamol possui como ação farmacológica, toxicidade, conheçam medidas de administração de antídotos e, acima de tudo, promovam atitudes que induzam o uso racional de medicamentos. Tais medidas são extremamente importantes para evitar problemas como este.
\end{abstract}

PALAVRAS-CHAVE: Paracetamol. Acetominofem. Fatores de risco. Toxicidade.

Farmacêutica Residente em Saúde da Criança- Residência Multiprofissional em Saúde dal Criança - REMUSC. Hospital Infantil Arlinda Marques. ORCID: 0000-0002-6682-5588 Autor correspondente: lucianavilar.farma@hotmail.com Farmacêutica Residente em Saúde da Criança- Residência Multiprofissional em Saúde da " Criança - REMUSC. Hospital Infantil Arlinda Marques. ORCID: 0000-0002-1509-2652 Professor da Faculdade de Medicina Nova Esperança - FAMENE. Farmacêutico e Tutor da III REMUSC. ORCID: 0000-0002-0824-4056 Professora da Faculdade de Enfermagem Nova Esperança- FACENE. Farmacêutica e $\mathrm{e}^{\mathrm{IV}}$ Preceptora da REMUSC. ORCID: 0000-0002-6498-0453 


\section{INTRODUÇÃO}

Inúmeras são as formas de intoxicação existentes, sejam por agrotóxicos, plantas, animais peçonhentos, entre outras. No entanto, as intoxicações por medicamentos ocupam o topo desta lista, sendo a mais comum em crianças e adolescentes de o a 15 anos. Devido à facilidade de acesso aos medicamentos, além do excesso de propagandas que induzem cada vez mais o consumo, as intoxicações se tornaram cada vez mais comuns, decorrentes, inúmeras vezes, do uso irracional. Os anti-inflamatórios não esteroidais (AINE's) perfazem uma classe de medicamentos bastante presente, em casos de intoxicação, destacando-se o paracetamol como o mais relevante, devido suas características hepatotóxicas. ${ }^{1,2}$

A toxicidade por acetaminofeno é um dos principais agentes de insuficiência hepática aguda nos Estados Unidos. Enquanto muitos casos têm uma história definida de exposição ao acetaminofeno, há um número considerável de ocorrências em que a causa da lesão hepática não é clara. 3

O paracetamol, também conhecido como acetominofeno, é um derivado do $\mathrm{N}$-acetil-P-aminofenol e foi desenvolvido em 1852 para fins analgésicos e antitérmicos. No entanto, apenas no ano de 1951 ele foi aprovado para uso e hoje se configura como um dos medicamentos mais utilizados, com ou sem prescrição médica, tendo média ação na analgesia, alta ação contra a febre e baixa ação anti-inflamatória quando comparado com os demais AINES. 4,5

O paracetamol é um medicamento de venda livre que se apresenta na forma sólida (comprimido, drágeas, cápsulas, pós e pastiIhas), ou líquida (gotas, solução e xarope), sozinho ou em associação. Todavia, como consequência da fácil aquisição, bem como do pouco conhecimento sobre os efeitos prejudiciais ao organismo, tem crescido o número de intoxicações em crianças. ${ }^{6}$

As pesquisas demonstram que os AlNES são o grupo farmacológico mais envolvido na prática da automedicação. São fármacos eficientes no tratamento da dor relacionada à inflamação e a lesão tecidual, a exemplo da artrite reumatoide e osteoartrite. 7

Sabe-se que a automedicação pode levar a intoxicação. No caso do paracetamol, aumentam os estudos que comprovam toxicidade e menor eficácia quando comparados à dipirona e ao ibuprofeno, somado ao fato desses medicamentos serem de venda livre, existe uma significância na intoxicação, principalmente em crianças menores. ${ }^{8}$

Nos Estados Unidos e Inglaterra, o paracetamol é a principal causa de falência hepática, seja por superdosagem provocada ou intencional, ou por ser também o principal fármaco envolvido em óbitos relatados nos centros de intoxicação nos EUA, nos anos 90.9

O quadro clínico de intoxicação com este fármaco revela acontecimentos bem definidos, tais como: nas primeiras 24 horas o paciente apresenta-se sem sintomas, ou no máximo com leve mal-estar, náuseas e epigastralgia. Em até 72 horas apresenta dor em hipocôndrio direito. Sua modificação mais característica é a elevação de transaminases hepáticas. Na fase de insuficiência hepática aguda, se não houver óbito, a reversibilidade é total, pois o tecido hepático consegue regenerar-se por completo entre sete dias até duas semanas. Destaca-se que, quando há o risco de hepatotoxicidade, é indicado o uso de acetilcisteína, antídoto específico nos casos de intoxicação por paracetamol. 4

Assim, faz-se importante compreender as características destas intoxicações bem como os fatores predisponentes para ocorrência destas, considerando a facilidade de acesso 
a estes medicamentos, bem como o perfil de automedicação que a sociedade atual apresenta.

Diante dessas informações preliminares, este estudo tem como objetivo investigar

\section{MATERIAL E MÉTODOS}

Trata-se de um estudo de revisão bibliográfica de caráter exploratório e descritivo, através de pesquisa em livros e artigos científicos, realizada durante o período de juIho e dezembro de 2017.

A exposição das palavras-chave foi feita consultando o Descritor em Ciências da Saúde (DeCS), através do site: http://decs.bvs. br A busca de artigos foi efetuada nas bases eletrônicas de dados Scielo, Science Direct, PubMed, Lilacs e Google Acadêmico. Foram considerados como critérios de inclusão para essas fontes: artigos publicados durante os anos de 2011 a 2017, artigos que abordassem intoxicações por paracetamol e outros com assuntos relacionados direta ou indiretamente ao assunto tratado para compor a introdução. Como critérios de exclusão, foram descartados artigos que não se enquadravam aos objetivos propostos pelo trabalho, além

\section{RESULTADOS E DISCUSSÕES}

\section{Farmacocinética}

O Paracetamol tem boa disponibilidade por via oral e o pico de ligação às proteínas plasmáticas ocorre em 30-60 minutos. A meia-vida plasmática é de cerca de 2 horas. ${ }^{10}$

Este fármaco é um ácido fraco com um pKa $=9,5$ a $25^{\circ} \mathrm{C}$. Em meio alcalino é bastante não-ionizado, de maneira que é bem absorvido no intestino delgado, através de difusão passi- os principais fatores que predispõem a hepatotoxicidade do paracetamol e mecanismos responsáveis por esse efeito de toxidade, tendo em vista o seu perfil de utilização.

de trabalhos de conclusão de curso e/ou especialização, dissertações, teses e anais de congressos. As palavras-chave utilizadas nas buscas foram: "toxicidade", "paracetamol" e "acetaminofen". A busca dos artigos foi realizada nos idiomas português e inglês.

Foram selecionados 167 artigos para análise e seleção final. A leitura do título e resumo foi feita como estratégia para seleção. Porém, quando a leitura do título e resumo não foi suficiente, procedeu-se a leitura na íntegra da publicação. Foram excluídos 148 artigos que não eram adequados ao tema da pesquisa, ou não respondiam ao objetivo do estudo. Acrescenta-se que três teses também foram excluídas. Por fim, a seleção destes foi composta por 18 artigos, a partir de análise dos títulos, bem como do conteúdo dos resumos que deveriam seguir os critérios de inclusão. va. Já a absorção no estômago é ínfima, ocorrendo esvaziamento gástrico do paracetamol para o duodeno. ${ }^{11}$

No que diz respeito a sua metabolização, o fígado é o principal órgão envolvido neste processo e sabe-se que a metabolização do paracetamol ocorre por três mecanismos: glicuronidação, sulfatação e oxidação. A sulfatação é a principal via de conjugação em crianças 
e nos adultos é aglicuronidação, que é alcançado por volta dos 12 anos. Quando ocorre uma dose acima de $4 \mathrm{~g} /$ dia, há saturação das vias metabólicas principais, provocando pela oxidação a geração do metabólito tóxico $\mathrm{N}$-acetil-p-benzo-quinonaimina (NAPQI). ${ }^{12}$

Em adultos, a meia vida de eliminação do paracetamol é aproximadamente 2 a 3 horas e em crianças é cerca de 1:30 a 3 horas. Ela é cerca de uma hora mais longa em recém-nascidos e em pacientes com cirrose. ${ }^{13} \mathrm{O}$ paracetamol é eliminado do organismo sob a forma de conjugado glucoronídeo ( $45 \%$ a $60 \%$ ) e conjugado sulfato ( $25 \%$ a $35 \%$ ), tiois ( $5 \%$ a $10 \%$ ), como metabólitos de cisteína e mercaptopurato e catecois (3\% a 6\%), que são excretados na urina e sua depuração renal do inalterado é por volta de 3,5\% da dose. ${ }^{14}$

\section{cos e antitérmicos}

Farmacodinâmica dos efeitos analgési-

O paracetamol é considerado hepatotóxico dependente de dose podendo gerar lesão de hepatócitos, através de mecanismos independentes ou associados entre si, como: (1) overdose (ingestão superior a $10 \mathrm{~g}$ em adultos e até $150 \mathrm{mg} / \mathrm{kg}$ em crianças); (2) situação de excessiva ativação do citocromo P450 e (3) depleção dos níveis de glutationa do hepatócito. ${ }^{14}$

Encontra-se bem esclarecida a presença de uma terceira forma de cicloxigenase (COX), a COX-3, que é uma isoforma da COX-1, sendo também nomeada de COX 1-b, em que está bem determinada a ação inibitória preferencial do paracetamol sobre essa isoforma. 3

As cicloxigenases são enzimas que induzem a atividade de prostaglandinas, que por sua vez, sensibilizam nociceptores periféricos e induzem sintomas da inflamação. Além disso, sabe-se que essa enzima tem expressão majoritária no SNC, sugerindo que o paracetamol tem um efeito mais central do que periférico. Dessa maneira, este fármaco mostra grande efeito antitérmico e analgésico (ação central), ausên- cia de efeito anti-inflamatório e de efeitos colaterais comuns ao grupo, como lesão gástrica, já que não existe COX-3 em mucosa gástrica, apenas a isoforma COX1-a). ${ }^{15}$

\section{Farmacodinâmica dos efeitos tóxicos}

$\mathrm{Na}$ administração de doses tóxicas de paracetamol, as vias de glicuronidação e sulfatação que, por sua vez, estão envolvidos na biotransformação de fármacos, se saturam, formando mais NAPQI, de maneira que as reservas de glutationa hepática se esgotam também e a reação com os grupos sulfídricos das proteínas se eleva bloqueando o fluxo de cálcio e levando, portanto, a necrose de hepatócitos.7

\section{Fatores que predispõem à hepatotoxi- cidade pelo paracetamol}

Nesta perspectiva, é importante considerar que, além da dose e tempo de utilização, existem fatores que podem tornar o indivíduo susceptível a hepatotoxidade por este medicamento, como idade, estado nutricional, ingestão crônica de álcool, tabagismo, fatores genéticos e associação a outros medicamentos. 5

Em relação ao estado nutricional, o metabolismo do acetaminofeno pode ser alterado sob condições que influenciam os estoques de glutationa. Obesidade, esteatose hepática, fome e jejum levam ao esgotamento de GSH e podem ser considerados como condição de risco para hepatotoxicidade, induzida por acetaminofeno, e um jejum sustentado resulta no redirecionamento do metabolismo do acetaminofeno da glucuronidação para a via de oxidação. Em condições de jejum, o metabolismo hepático é desviado para a gliconeogênese, convertendo menos precursores da glicose disponíveis para a glucuronidação. ${ }^{16}$

Medicamentos como fenobarbital que induzem a atividade do citocromo P450 (CYP) podem elevar os níveis de NAPQI favorecendo a ocorrência de hepatotoxicidade. No jejum prolongado, assim como no etilismo crônico, ocorre redução nos níveis de glutationa induzindo o CYP 2E1 elevando assim a toxicidade 
para o fígado. O tabagismo também é um fator importante a ser considerado uma vez que o tabaco contém em sua composição substâncias que induzem CYP1A2 acentuando o metabolismo oxidativo e, por sua vez, a hepatotoxicidade. 5

Polimorfismos nas isoenzimas do CYP também podem conferir metabolização exacerbada por determinados compostos. Consequências na variação dessas formas de enzimas incluem alteração cinética e ação de determinadas drogas, reações adversas idiossicrásicas e interações medicamentosas. Por fim, a idade também é um fator importante a ser considerado. Como em adultos a metabolização por CYP é mais pronunciada, a hepatotoxicidade pode ocorrer em doses menores, ao passo que em crianças a metabolização ocorre mais acentuadamente pelas vias de sulfatação e glicuronização. Indivíduos portadores de hepatopatias também são mais susceptíveis a ocorrência de hepatotoxicidade por paracetamol.5,17,9

\section{Tratamento da intoxicação}

Existem muitas ações diferentes que podem ser utilizadas para tentar tratar pessoas com intoxicação por paracetamol. Estas intervenções visam diminuir a absorção do paracetamol ingerido e, assim, reduzir a quantidade

\section{CONSIDERAÇÕES FINAIS}

Diante do exposto acima, é importante que os profissionais de saúde conheçam as características farmacológicas desse medicamento, os efeitos de sua toxicidade, os fatores que predispõem, bem como, as ações a serem realizadas nos casos de urgências envolvendo overdose por paracetamol. Este medicamento é considerado relativamente seguro quan- absorvida na corrente sanguínea. Os agentes incluem carvão ativado, que se liga ao paracetamol no estômago e lavagem gástrica. Nos casos de intoxicação é recomendada a lavagem gástrica, procedimento comum em casos de overdoses. Esta deverá ser realizada em até duas horas após o evento, de modo que a absorção do paracetamol seja reduzida. Pode-se usar ainda, o carvão ativado que irá reduzir a absorção no trato gastrintestinal, além da acetilcisteína que é um antídoto bastante eficaz, capaz de neutralizar o metabólito tóxico, fazendo com que não ocorra dano hepático. ${ }^{18,5}$

A acetilcisteína atua na hepatotoxicidade restaurando as reservas de glutationa hepática, através do fornecimento de cisteína, constituindo-se na chave para a eficácia, quando administrada nas primeiras oito horas da ingestão aguda de paracetamol. ${ }^{13}$

A administração se dá por via oral ou endovenosa. Na primeira, por via nasogástrica, faz-se $140 \mathrm{mg} / \mathrm{kg}$, seguido de $70 \mathrm{mg} / \mathrm{kg}$ de quatro em quatro horas que totalize 17 administrações. Já na via intravenosa, administra-se uma dose de impregnação de $140-150 \mathrm{mg} / \mathrm{kg}$ em dextrose a $5 \%$ correndo em 15 minutos, seguido de $70 \mathrm{mg} / \mathrm{kg}$ de quatro em quatro horas também num total de 17 administrações. 5 do usado em posologia e dose adequadas. No entanto, devido ao fato de ser isento de prescrição, possuir baixo preço e fácil acesso, tem propiciado aumento relevante de casos de intoxicação. Atitudes que visam promoção do uso racional de medicamentos são de extrema importância para evitar e minimizar problemas como este. 


\title{
AGRADECIMENTO
}

A João Elson Inocêncio pela revisão idiomática.

\section{PARACHETAMOL HEPATOTOXICITY AND PREDISPONENT FACTORS}

\begin{abstract}
The non-opioid analgesics don't constitute a remedy class very present in cases of intoxication being the acetaminophen (paracetamol), the most relevant due to its hepatotoxicity characteristics. For being an over-the-counter medication, it is easy to purchase and the little knowledge about its harmful effects to the organism contribute to the increase of intoxication mainly in children. Depending on the dose the paracetamol is considered hepatotoxicity and could cause lesion to the hepatocytes. The objective of this study is to investigate the main factors that predispose to hepatotoxicity of paracetamol and its mechanism of hepatotoxicity, considering its profile of use by means of an exploratory and descriptive bibliographic review through research in books and scientific articles carried out during the period of July and December 2017. The search of articles was made in the bases of data such as Scielo, Science Direct, PubMed, Lilacs and Google Academy. In the search for results, the articles were evaluated through the analysis of titles and abstracts and, in total, the analysis consisted of 19 articles. Some factors may predispose a person to paracetamol hepatotoxicity as drugs that inhibit enzymes, prolonged fasting, alcoholism, gene polymorphism, age, among others. It is important that the professionals involved with medical products known the characteristics that the paracetamol possesses as a pharmacological action, toxicity, predisposition to this event and measures of administration of antidotes and above all to promote attitudes the encourage careful use of medicines is extremely important to avoid problems.
\end{abstract}

KEYWORDS: Paracetamol. Acetominophen. Risk factors. Toxicity.

\section{REFERÊNCIAS}

1. Witter AA, Medeiros AISR, Teixeira LM, Barbosa MGM, Santos SP, Marques RB.Intoxicação medicamentosa em crianças: uma revisão de literatura. Revinter, 2016; 9 (3): 64-71.

2. Feldkircher KCG. Intoxicação medicamentosa em animais peçonhentos. Revista Científica de Medicina Veterinária, 2014; 1 (1):14-18.

3. Heard KJ, Green JL, James LP, Judge BS, Zolot
L, Rhyee S, Dart RC. Acetaminophen-cysteine adducts during therapeutic dosing and following overdose. Bio Med Central Gastroenterology, 2011; 11 (20).

4. Muhlbauer M. Paracetamol, um AINE particular. Ciência Atual, 2016; 7 (1): 2-10.

5. Terres DR. Potencial toxicológico de medicamentos de venda livre: ênfase no paracetamol. 
FACIDER Revista Científica, 2015; (8): 1-15.

6.Lopes J, Matheus ME. Risco de hepatoxicidade do paracetamol (Acetaminofem). Revista Brasileira de Farmácia, 2012; 93 (4): 411-414.

7. Sebben VC, Lugoch RW, Schilinker CS, Arbo MD, Vianna RL. Validação de metodologia para quantificação sérica de paracetamol. Jornal Brasileiro de Patologia Médica Laboratorial, 2010; 46 (2):143-148.

8. Vieira AL, França GG. As consequências no consumo indiscriminado do paracetamol e orientação farmacêutica à promoção ao uso racional. Revista Acadêmica Oswaldo, 2015; 30 (6): p. 1-12.

9. Uzam CPP, Paiva A, Viana D, Martins G, Molina N. Impacto dos medicamentos na intoxicação em crianças. Revista da Universidade Ibirapuera, 2017; (13): 8-16.

10. Silva GH, Barros PP, Gonçalves MS, Jesus EA. Avaliação da atividade hepatoprotetora do asiaticosídeo em modelo experimental de lesão hepática por paracetamol em ratos. Revista de Ciências Farmacêuticas Básica e Aplicada, 2014; 35 (3): 489-496.

11. Goodman; Gilman. As bases farmacológicas da terapêutica. 12 ed. Rio de Janeiro: McGraw-Hill, 2012.

12. Marzuillo P, Guarino S, Barbi E. Paracetamol: a focus for the general pediatrician. European Journal of Pediatrics, 2014; 173 (4):415-425;

13. Jiang XL, Zhao P,Barret JS, Lesko $\sqcup$, Schimi- dt S. Application of Physiolically based pharmacokinetic modeling to predict acetaminophen metabolism and pharmacokinetcs in children. OPT: Pharmacometrics \& Sistems Pharmacology, 2013, 2013; 2 (80): 1-9.

14. Paracetamol. Minas Gerais :Hipolabor farmacêutica.

15. Silva RNF, Pereira LCG. O uso de antiinflamatórios esteroidais e não esteroidais no controle da dor e do edema em cirurgia de terceiros molares. Revista Bahiana de Odontologia, 2016; 7(1): 31-39.

16. Mazaleuskaya LL, Sangkuhl K, Thorn CF, GA FitzGerald, Altman RB, Klein TE. PharmGKB summary: Pathways of acetaminophen metabolism at the therapeutic versus toxic doses. Pharmacogenet Genomics.. 2015; 25 (8): 416-26.

17. Junior EVM. Situações que favorecem ou reduzem a hepatotoxicidade pelo acetominofem (uso de álcool, faixa etária e uso de outras medicações). Revistas Suplemento hepatotoxicidade, 2011; 30 (suplemento 1) : 6-47.

18. Torres LV. Magnitude do polimorfismo nos genes da família do citocromo p450 nas ciências farmacêuticas: uma revisão de literatura. Revista de Ciências da Saúde Nova Esperança, 2016; 14 (especial):10-17.

19. Chiew AL, Gluud C, Brok J, Buckley NA. Interventions for paracetamol (acetaminophen) overdose. Cochrane Database of Systematic Reviews 2018, Issue 2. 